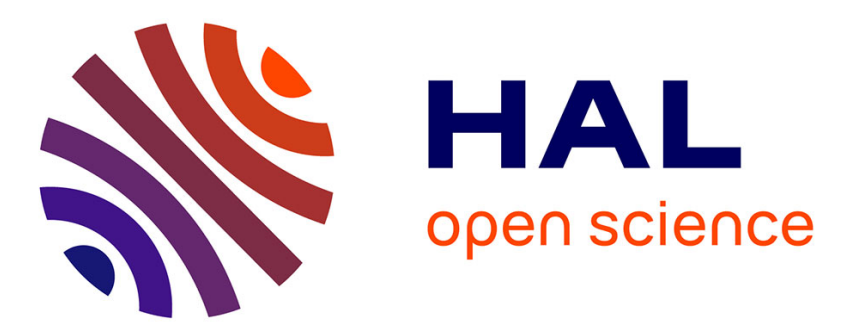

\title{
Nonlinear dynamics of nanomechanical beam resonators: improving the performance of NEMS-based sensors
}

\author{
Najib Kacem, Sébastien Hentz, David Pinto, Bruno Reig, V.S. Nguyen
}

\section{To cite this version:}

Najib Kacem, Sébastien Hentz, David Pinto, Bruno Reig, V.S. Nguyen. Nonlinear dynamics of nanomechanical beam resonators: improving the performance of NEMS-based sensors. Nanotechnology, 2010, 20 (27), 10.1088/0957-4484/20/27/275501 . hal-00505123

\section{HAL Id: hal-00505123 \\ https://hal.science/hal-00505123}

Submitted on 20 Jan 2018

HAL is a multi-disciplinary open access archive for the deposit and dissemination of scientific research documents, whether they are published or not. The documents may come from teaching and research institutions in France or abroad, or from public or private research centers.
L'archive ouverte pluridisciplinaire HAL, est destinée au dépôt et à la diffusion de documents scientifiques de niveau recherche, publiés ou non, émanant des établissements d'enseignement et de recherche français ou étrangers, des laboratoires publics ou privés. 


\title{
Nonlinear dynamics of nanomechanical beam resonators: improving the performance of NEMS-based sensors
}

\author{
N Kacem, S Hentz, D Pinto, B Reig and V Nguyen \\ CEA/LETI—MINATEC, Grenoble, France
}

\begin{abstract}
In order to compensate for the loss of performance when scaling resonant sensors down to NEMS, it proves extremely useful to study the behavior of resonators up to very high displacements and hence high nonlinearities. This work describes a comprehensive nonlinear multiphysics model based on the Euler-Bernoulli equation which includes both mechanical and electrostatic nonlinearities valid up to displacements comparable to the gap in the case of an electrostatically actuated doubly clamped beam. Moreover, the model takes into account the fringing field effects, significant for thin resonators. The model has been compared to both numerical integrations and electrical measurements of devices fabricated on $200 \mathrm{~mm}$ SOI wafers; it shows very good agreement with both. An important contribution of this work is the provision for closed-form expressions of the critical amplitude and the pull-in domain initiation amplitude including all nonlinearities. This model allows designers to cancel out nonlinearities by tuning some design parameters and thus gives the possibility to drive the resonator beyond its critical amplitude. Consequently, the sensor performance can be enhanced to the maximum below the pull-in instability, while keeping a linear behavior.
\end{abstract}

\section{Introduction}

Nanoelectromechanical systems (NEMS) have been the focus of recent applied and fundamental research. With critical dimensions down to a few tens of nanometers, most NEMS are used working in resonance. In this size regime they display high fundamental resonance frequencies (recently going beyond $1 \mathrm{GHz}[1,2])$, diminished active masses, tolerable force constants and relatively high quality factors in the range of $10^{2}-10^{4}$. These attributes collectively make NEMS suitable for a multitude of technological applications such as ultrasensitive force and mass sensing, narrow band filtering and time keeping. So as to fulfil their full promise, that is, to begin to come out of industrial foundries, a certain number of challenges are yet to be addressed: in particular, their frequency stability, i.e. their output carrier power, has to be improved. Besides, the smaller the structures, the sooner nonlinearities occur [3], reducing their dynamic range and even making it extremely difficult to detect their oscillation, as the abundant literature about characterization techniques proves [4].
The resolution of a resonant sensor may be given by its frequency noise spectral density [5]:

$$
S_{\omega}(\omega)=\left(\frac{\omega_{n}}{2 Q}\right)^{2} \frac{S_{x}(\omega)}{P_{0}}
$$

where $S_{x}(\omega)$ is the displacement spectral density and $P_{0}$ is the displacement carrier power, i.e. the RMS drive amplitude of the resonator $\frac{1}{2} A^{2}$. Following Postma and Roukes [3], the resonator critical amplitude is $A_{\mathrm{c}} \propto \frac{h}{Q}$, where $h$ is the resonator thickness in the direction of vibration and $Q$ is its quality factor. It corresponds to the hysteretic limit below which the resonator is classically driven due to the mechanical nonlinearity. It is easy to see how drastic the performance degradation may be in the case of a NEMS with small $h$. It has been shown that closed-loop control allows operation beyond the critical amplitude [6], eventually up to the pull-in amplitude in the case of capacitive transduction. But to do so, it is necessary first to precisely know up to which amplitude the resonator may be driven, and second to avoid the noise aliasing issue [7], so as not to degrade the amplitude noise density. 


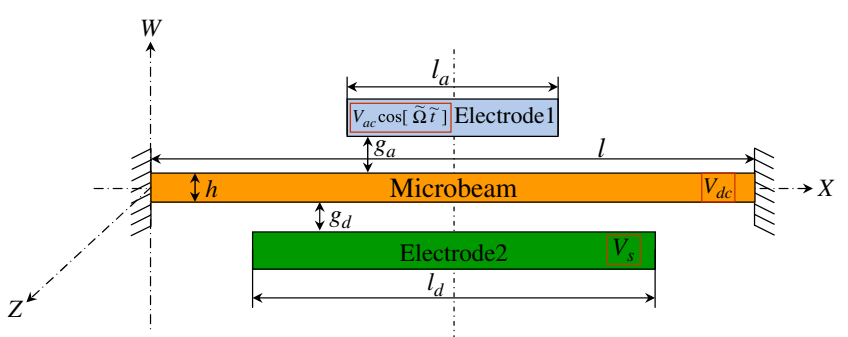

Figure 1. Schema of an electrically actuated microbeam.

To this end, the nonlinear behavior of resonators remains yet to be explored, and numerous models have been presented. Some of them are purely analytical [8-10], but they include coarse assumptions concerning nonlinearities. For example, Kozinsky et al [10] use a nonlinear model with a third order Taylor series expansion of the electrostatic forcing applied to a nanoresonator in order to tune the effective Duffing coefficient using an external electrostatic potential. However, this approximation is limited by the beam displacement (no more than $20 \%$ of the gap). Moreover, the static displacement is used for tuning, which stays limited for practical designs of resonators with high quality factors because of the very small ratio between the static and the dynamic displacement, which makes the nonlinear coupling between both components negligible.

Other models are more complicated and use numerical integrations such as differential quadrature method in [11] and shooting in [12]. These methods are computationally more demanding, which makes them less interesting for M/NEMS designers. Osterberg and Senturia [13] use the finite difference method to determine the static pull-in parameters and provide approximate empirical formulae. In [14] the pull-in instability is studied using the shooting method, and in [15] reducedorder models and perturbation techniques are used to analyze the pull-in behavior without giving a closed-form solution. In the present paper, a compact and analytical model including all main sources of nonlinearities is presented and validated thanks to the characterization of a vibrating accelerometer sensing element, an electrostatically driven clamped-clamped beam. It is shown how the model can be used to engineer the resonator nonlinearities in order to drive it at the highest amplitude possible while suppressing its hysteresis, thus retrieving a linear behavior. Moreover, a bifurcation point criterion was used, providing closed-form solutions of the critical amplitude and the pull-in domain initiation voltage.

\section{Model}

A clamped-clamped microbeam is considered (figure 1) subject to a viscous damping with coefficient $\tilde{c}$ per unit length and actuated by an electric load $v(t)=-V_{\mathrm{dc}}+V_{\mathrm{ac}} \cos (\tilde{\Omega} \tilde{t})$, where $V_{\mathrm{dc}}$ is the DC polarization voltage, $V_{\mathrm{ac}}$ is the amplitude of the applied AC voltage, and $\tilde{\Omega}$ is the excitation frequency.

Existing models [11, 12] for the nonlinear dynamics of MEMS resonators with relatively high capacitive variations concern designs with one electrode for actuation and sensing.
NEMS resonators, though, have low capacitive variations, and it is almost essential to use a two port measurement, i.e. to separate detection and actuation electrodes. Moreover, the use of different gaps $\left(g_{\mathrm{d}}<g_{\mathrm{a}}\right)$ enables the maximization of the detection signal. Below, we detail the equation of motion, showing the complexity of the PDE to be solved.

\subsection{Equation of motion}

The transverse deflection of the microbeam $\tilde{w}(x, t)$ is governed by the nonlinear Euler-Bernoulli equation, which is the commonly used approximate equation of motion for a thin beam [16].

$$
\begin{aligned}
& E I \frac{\partial^{4} \tilde{w}(\tilde{x}, \tilde{t})}{\partial \tilde{x}^{4}}+\rho b h \frac{\partial^{2} \tilde{w}(\tilde{x}, \tilde{t})}{\partial \tilde{t}^{2}}+\tilde{c} \frac{\partial \tilde{w}(\tilde{x}, \tilde{t})}{\partial \tilde{t}} \\
& \quad-\left[\tilde{N}+\frac{E b h}{2 l} \int_{0}^{l}\left[\frac{\partial \tilde{w}(\tilde{x}, \tilde{t})}{\partial \tilde{x}}\right]^{2} \mathrm{~d} \tilde{x} \frac{\partial^{2} \tilde{w}(\tilde{x}, \tilde{t})}{\partial \tilde{x}^{2}}\right. \\
& =\frac{1}{2} \varepsilon_{0} \frac{b C_{n 1}\left[V_{\mathrm{ac}} \cos (\tilde{\Omega} \tilde{t})-V_{\mathrm{dc}}\right]^{2}}{\left(g_{\mathrm{a}}-\tilde{w}(\tilde{x}, \tilde{t})\right)^{2}} H_{1}(\tilde{x}) \\
& \quad-\frac{1}{2} \varepsilon_{0} \frac{b C_{n 2}\left[V_{\mathrm{s}}-V_{\mathrm{dc}}\right]^{2}}{\left(g_{\mathrm{d}}+\tilde{w}(\tilde{x}, \tilde{t})\right)^{2}} H_{2}(\tilde{x}) \\
& H_{1}(\tilde{x})=H\left(\tilde{x}-\frac{l+l_{\mathrm{a}}}{2}\right)-H\left(\tilde{x}-\frac{l-l_{\mathrm{a}}}{2}\right) \\
& H_{2}(\tilde{x})=H\left(\tilde{x}-\frac{l+l_{\mathrm{d}}}{2}\right)-H\left(\tilde{x}-\frac{l-l_{\mathrm{d}}}{2}\right)
\end{aligned}
$$

where $\tilde{x}$ is the position along the microbeam length, $b$ is the beam width in the out-of-plane direction $Z$, and $E$ and $I$ are the Young's modulus and moment of inertia of the cross section. $\tilde{N}$ is the applied tensile axial force due to the residual stress on the beam material, $\tilde{t}$ is time, $\rho$ is the material density, $h$ is the microbeam thickness, $g_{\mathrm{a}}$ and $g_{\mathrm{d}}$ are respectively the actuation and the sensing capacitor gap width, and $\varepsilon_{0}$ is the dielectric constant of the gap medium. The last term in equation $(2 a)$ represents an approximation of the electric force assuming a resonator design with two stationary electrodes: electrode 1 for the actuation and electrode 2 for the sensing including the fringing field effect [17], using the coefficients $C_{n i}$ (see section 2.8). $H(\tilde{x})$ are Heaviside functions modeling the electrostatic force distributions. The boundary conditions are

$$
\begin{gathered}
\tilde{w}(0, \tilde{t})=\tilde{w}(l, \tilde{t})=0 \\
\frac{\partial \tilde{w}}{\partial \tilde{x}}(0, \tilde{t})=\frac{\partial \tilde{w}}{\partial \tilde{x}}(l, \tilde{t})=0 .
\end{gathered}
$$

\subsection{Normalization}

For convenience and equation simplicity, we introduce the nondimensional variables:

$$
w=\frac{\tilde{w}}{g_{\mathrm{d}}}, \quad x=\frac{\tilde{x}}{l}, \quad t=\frac{\tilde{t}}{\tau}
$$

where $\tau=\frac{2 l^{2}}{h} \quad \overline{\frac{3 \rho}{E}}$. 
Substituting equation (4) into equations (2a), (3a) and $(3 b)$, we obtain

$$
\begin{gathered}
\frac{\partial^{4} w}{\partial x^{4}}+\frac{\partial^{2} w}{\partial t^{2}}+c \frac{\partial w}{\partial t}+\alpha_{2} C_{n 2} \frac{\left[V_{\mathrm{s}}-V_{\mathrm{dc}}\right]^{2}}{(1+w)^{2}} H_{2}(x) \\
=\alpha_{2} C_{n 1} \frac{\left[V_{\mathrm{ac}} \cos (\Omega t)-V_{\mathrm{dc}}\right]^{2}}{\left(R_{\mathrm{g}}-w\right)^{2}} H_{1}(x) \\
+\left[N+\alpha_{1} \int_{0}^{1}\left[\frac{\partial w}{\partial x}\right]^{2} \mathrm{~d} x\right] \frac{\partial^{2} w}{\partial x^{2}} \\
w(0, t)=w(1, t)=\frac{\partial w}{\partial x}(0, t)=\frac{\partial w}{\partial x}(1, t)=0 .
\end{gathered}
$$

The parameters appearing in equation $(5 a)$ are

$$
\begin{aligned}
& H_{2}(x)=H\left(x-\frac{l+l_{\mathrm{a}}}{2 l}\right)-H\left(x-\frac{l-l_{\mathrm{a}}}{2 l}\right) \\
& H_{2}(x)=H\left(x-\frac{l+l_{\mathrm{d}}}{2 l}\right)-H\left(x-\frac{l-l_{\mathrm{d}}}{2 l}\right) \\
& \left.c=\frac{\tilde{c} l^{4}}{E I \tau}, \quad \quad N=\frac{\tilde{N} l^{2}}{E I}, \quad \alpha_{1}=6 \frac{g_{\mathrm{a}}}{h}\right]^{2} \\
& R_{\mathrm{g}}=\frac{g_{\mathrm{a}}}{g_{\mathrm{d}}}, \quad \alpha_{2}=6 \frac{\varepsilon_{0} l^{4}}{E h^{3} g_{\mathrm{a}}^{3}}, \quad \Omega=\tilde{\Omega} \tau .
\end{aligned}
$$

\subsection{Solving}

The beam total displacement $w(x, t)$ can be written as a sum of a static de displacement $w_{\mathrm{s}}(x)$ and a time-varying ac displacement $w_{\mathrm{d}}(x, t)$. However, for our devices, it is easy to check that the static deflection is negligible. Typically, the measured quality factors $Q$ are in the range of $10^{4}-5 \times 10^{4}$ and the $V_{\mathrm{dc}} \leqslant 200 V_{\mathrm{ac}}$. Thus, the ratio between the static and the dynamic deflection is

$$
\frac{w_{\mathrm{s}}(x)}{w_{\mathrm{d}}(x, t)} \approx \frac{V_{\mathrm{dc}}}{2 Q V_{\mathrm{ac}}} \leqslant 1 \% .
$$

A reduced-order model is generated by modal decomposition transforming equation ( $5 a$ ) into a finite-degree-of-freedom system consisting in ordinary differential equations in time. We use the undamped linear mode shapes of the straight microbeam as basis functions in the Galerkin procedure. To this end, we express the deflection as

$$
w(x, t)={ }_{k=1}^{n} a_{k}(t) \phi_{k}(x)
$$

where $a_{k}(t)$ is the $k$ th generalized coordinate and $\phi_{k}(x)$ is the $k$ th linear undamped mode shape of the straight microbeam, normalized such that $\int_{0}^{1} \phi_{k} \phi_{j}=\delta$ where $\delta=0$ if $k \neq j$ and $\delta=1$ if $k=j$. The linear undamped mode shape $\phi_{k}(x)$ is governed by

$$
\begin{gathered}
\frac{\mathrm{d}^{4} \phi_{k}(x)}{\mathrm{d} x^{4}}=\lambda_{k}^{2} \phi_{k}(x) \\
\phi_{k}(0)=\phi_{k}(1)=\phi_{k}^{\prime}(0)=\phi_{k}^{\prime}(1) .
\end{gathered}
$$

Here, $\lambda_{k}$ is the $k$ th natural frequency of the microbeam. We multiply equation $(5 a)$ by $\phi_{k}(x)\left[(1+w)\left(R_{\mathrm{g}}-w\right)\right]^{2}$ in order to include the complete contribution of the nonlinear electrostatic forces in the resonator dynamics without approximation. This particular step, similar to what Nayfeh et al [12] used but with only one electrode, increases the level of nonlinearity in the normalized equation of motion $(5 a)$.

We substitute equation (8) into the resulting equation, use equation (9) to eliminate $\frac{\mathrm{d}^{4} \phi_{k}(x)}{\mathrm{d} x^{4}}$, integrate the outcome from $x=0$ to 1 , and obtain a system of coupled ordinary differential equations in time.

Assuming that the first mode should be the dominant mode of the system and the other modes are neglected (assumption discussed later: see section 2.7), it is enough to consider $n=1$ and obtain

$$
\begin{aligned}
\ddot{a}_{1}+ & c \dot{a}_{1}+\omega_{n}^{2} a_{1}+\mu_{1} a_{1} \ddot{a}_{1}+\mu_{2} a_{1}^{2} \ddot{a}_{1}+\mu_{3} a_{1}^{3} \ddot{a}_{1} \\
& +\mu_{4} a_{1}^{4} \ddot{a}_{1}+c \mu_{1} a_{1} \dot{a}_{1}+c \mu_{2} a_{1}^{2} \dot{a}_{1}+c \mu_{3} a_{1}^{3} \dot{a}_{1} \\
& +c \mu_{4} a_{1}^{4} \dot{a}_{1}+\chi_{2} a_{1}^{2}+\chi_{3} a_{1}^{3}+\chi_{4} a_{1}^{4}+\chi_{5} a_{1}^{5} \\
& +\chi_{6} a_{1}^{6}+\chi_{7} a_{1}^{7}+v+\zeta_{0} \cos (\Omega t) \\
& +\zeta_{1} a_{1} \cos (\Omega t)+\zeta_{2} a_{1}^{2} \cos (\Omega t) \\
& +\zeta_{3} \cos (2 \Omega t)+\zeta_{4} a_{1} \cos (2 \Omega t) \\
& +\zeta_{5} a_{1}^{2} \cos (2 \Omega t)=0 .
\end{aligned}
$$

We recognize in equation (11) some canonical nonlinear terms such as the cubic stiffness term (Duffing nonlinearity) and the nonlinear Van der Pol damping $\left(c \mu_{2} a_{1}^{2} a_{1}^{\prime}\right)$ as well as the parametric excitation (Mathieu term). However, the presence of other high-level nonlinearities in equation (11) makes the described system in figure 3 a forced nonlinear resonator under multifrequency parametric excitation. This kind of equation is not so frequently used in the literature and includes terms coming from the coupling between the mechanical and the electrostatic nonlinearities as well as the nonlinear coupling between both electrostatic forces.

In the appendix, we show the expressions of all the integration parameters presented in equation (11), trivial to compute with any computational software. To analyze this equation of motion, we use perturbation techniques well adapted to 'small' excitation and damping $(Q>10)$, typically valid in NEMS resonators [18]. To facilitate the perturbation approach, in this case the method of averaging [19] for its ease of use, a standard constrained coordinate transformation is introduced, as given by

$$
\begin{gathered}
a_{1}=A(t) \cos [\Omega t+\beta(t)] \\
\dot{a}_{1}=-A(t) \Omega \sin [\Omega t+\beta(t)] \\
\ddot{a}_{1}=-A(t) \Omega^{2} \cos [\Omega t+\beta(t)] .
\end{gathered}
$$

In addition, since near-resonant behavior is the principal operating regime of the proposed system, a detuning parameter, $\sigma$, is introduced, as given by

$$
\Omega=\omega_{n}+\varepsilon \sigma
$$

Separating the resulting equations and averaging them over the period $\frac{2 \pi}{\Omega}$ in the $t$-domain results in the system's averaged equations, in terms of amplitude and phase, which are given by 

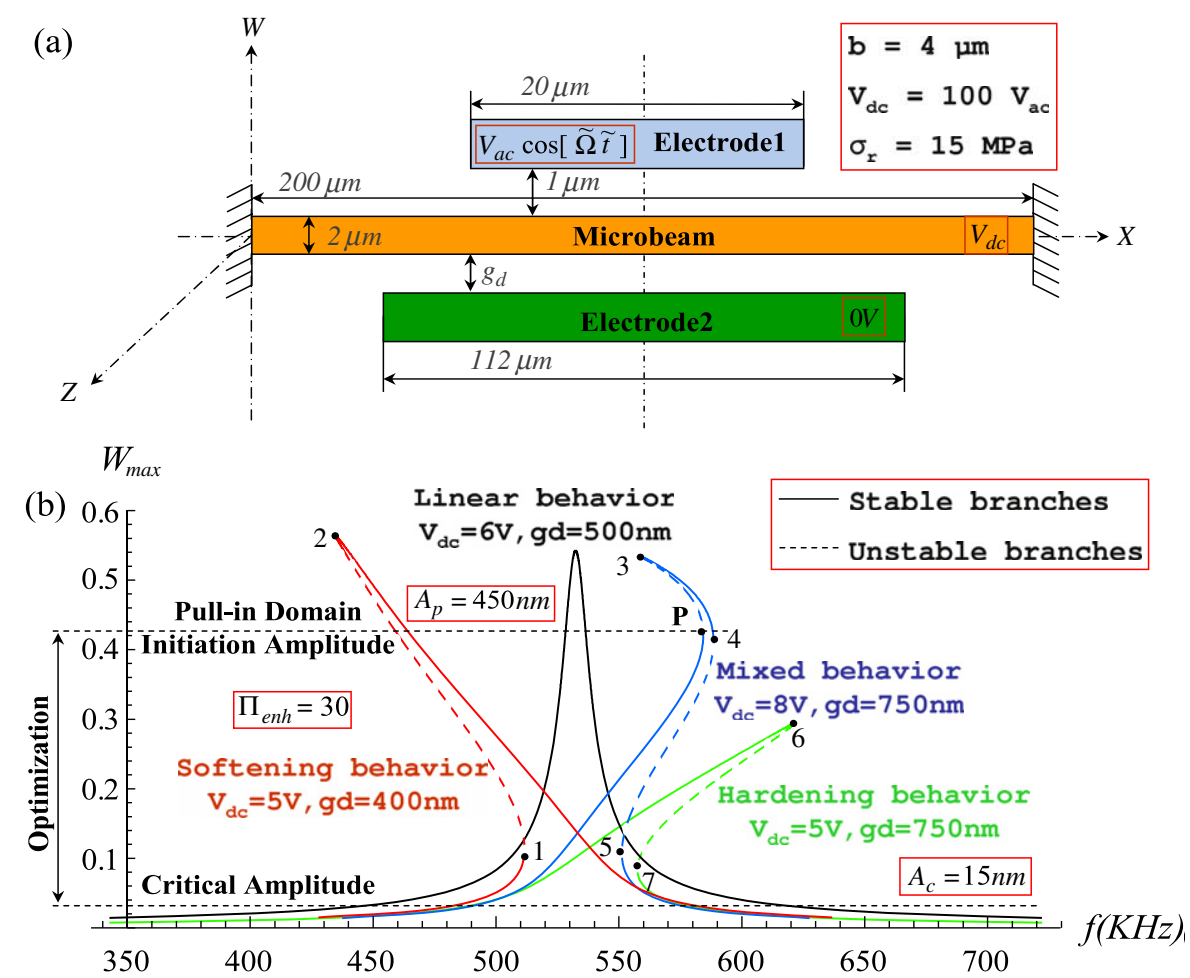

Figure 2. (a) Dimensions of a typical fabricated resonator. (b) Predicted forced frequency responses. $W_{\max }$ is the displacement of the beam normalized by the gap $g_{\mathrm{d}}$ at its middle point $\frac{l}{2}, \sigma_{\mathrm{r}}$ is the axial residual stress on the beam material, $A_{\mathrm{c}}$ is the critical amplitude above which bistability occurs, $\{1,2,3,4,5,6,7, P\}$ are the different bifurcation points, $A_{\mathrm{p}}$ is the pull-in domain initiation amplitude and $\mathrm{P}$ is the third bifurcation point characterizing the initiation of the mixed behavior.

$$
\begin{aligned}
\dot{A}= & \varepsilon \frac{\sin [\beta] \zeta_{0}}{2 \omega_{n}}+\varepsilon \frac{A^{2} \sin [\beta] \zeta_{2}}{8 \omega_{n}}-\varepsilon \frac{A c}{2} \\
& -\varepsilon \frac{A^{3} c \mu_{2}}{8}-\varepsilon \frac{A^{5} c \mu_{4}}{16}+\mathrm{O}\left(\varepsilon^{2}\right) \\
\dot{\beta}= & \varepsilon \sigma-\varepsilon \frac{3 A^{2} \chi_{3}}{8 \omega_{n}}-\varepsilon \frac{5 A^{4} \chi_{5}}{16 \omega_{n}}-\varepsilon \frac{35 A^{6} \chi_{7}}{128 \omega_{n}} \\
& -\varepsilon \frac{\cos [\beta] \zeta_{0}}{2 A \omega_{n}}-\varepsilon \frac{3 A \cos [\beta] \zeta_{2}}{8 \omega_{n}}+\varepsilon \frac{3}{8} A^{2} \omega_{n} \mu_{2} \\
& +\varepsilon \frac{5}{16} A^{4} \omega_{n} \mu_{4}+\mathrm{O}\left(\varepsilon^{2}\right) .
\end{aligned}
$$

The steady-state motions occur when $\dot{A}=\dot{\beta}=0$, which corresponds to the singular points of equations $(14 a)$ and $(14 b)$. Thus, the frequency-response equation can be written in its parametric form $\left\{A=K_{1}(\beta), \Omega=K_{2}(\beta)\right\}$ as a function of the phase $\beta$ as a set of two equations easy to introduce in Matlab or Mathematica. This ability makes the model suitable for NEMS designers as a quick tool of resonant sensor performance optimization.

For the sake of clarity, the frequency-response equation can be written in its implicit form as

$$
\begin{aligned}
& \frac{A^{2}\left\{\Lambda_{1}(A)-8 \omega_{n}\left(16 \Omega+\Lambda_{2}(A)\right)\right\}^{2}}{256\left(4 \zeta_{0}+3 A^{2} \zeta_{2}\right)^{2}} \\
& \quad+\frac{\left(8 c A+2 A^{3} c \mu_{2}+A^{5} c \mu_{4}\right)^{2} \omega_{n}^{2}}{4\left(4 \zeta_{0}+A^{2} \zeta_{2}\right)^{2}}=1 \\
& \Lambda_{1}(A)=48 A^{2} \chi_{3}+40 A^{4} \chi_{5}+35 A^{6} \chi_{7} \\
& \Lambda_{2}(A)=\left(6 A^{2} \mu_{2}+5 A^{4} \mu_{4}-16\right) \omega_{n} .
\end{aligned}
$$
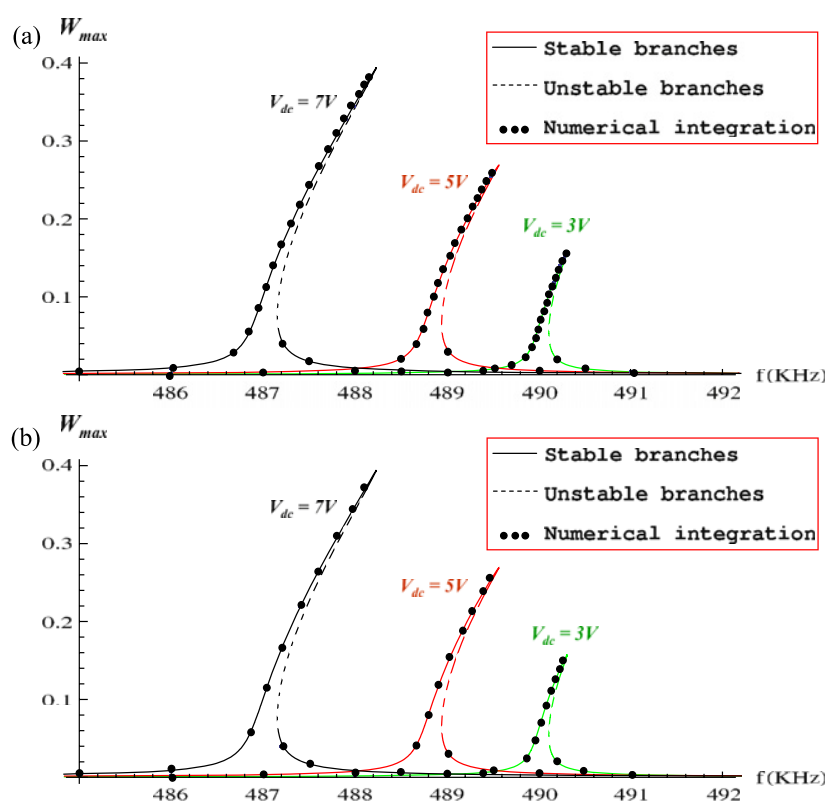

Figure 3. (a) Numerical validation with one mode. (b) Numerical validation with five modes. $W_{\max }$ is the displacement of the beam normalized by the gap $g_{\mathrm{d}}$ at its middle point $\frac{l}{2}$. When the DC voltage increases, the negative electrostatic stiffness increases and shifts the natural frequency of the resonator to low frequencies.

In particular, for one of our devices (figure 2(a)), the frequency-response curve can be plotted parametrically as shown in figure $2(\mathrm{~b})$. 
This analytical model enables the capture of all the nonlinear phenomena in the resonator dynamics and describes the competition between the hardening and the softening behaviors. In addition, the model allows the optimization of resonator designs by tuning the parameter $\frac{h}{g_{\mathrm{d}}}$ in order to cancel out nonlinearities as shown in figure 2(b) for $g_{\mathrm{d}}=500 \mathrm{~nm}$ (black curve), thus enhancing the limit of detection of NEMS resonant sensors as explained below.

\subsection{The critical amplitude}

The critical amplitude is the oscillation amplitude $A_{\mathrm{c}}$ above which bistability occurs. Thus, $A_{\mathrm{c}}$ is the transition amplitude from the linear to the nonlinear behavior.

At the critical drive, the resonance curve exhibits a point of infinite slope, called the critical point. Moreover, at the same point, the phase curve also exhibits an infinite slope at the same detuning as the resonance curve itself. Nayfeh studied the stability of an excited Duffing oscillator [20] and deduced its critical amplitude. Kaajakari [21] provided a closed-form expression for the critical amplitude using a reduced-order model including the crystalline direction of beam resonators. However, they do not incorporate in their models the complete contribution of the electrostatic nonlinearities.

In order to explain how to deduce the critical amplitude from equation (15a) written in its parametric form, we assume the simplified case of neglected nonlinear electrostatic effects $\left(\frac{h}{g_{\mathrm{d}}} \ll 1\right)$. The parametric form of the frequency response can be written as

$$
\begin{gathered}
\Omega=\frac{1}{8}\left(\frac{3 \kappa^{2} \gamma_{3}}{\xi_{0}^{2} \omega_{n}^{3}} \sin ^{2} \beta-4 \xi_{0} \cot \beta\right)+\omega_{n} \\
A=\frac{\kappa}{\xi_{0} \omega_{n}} \sin \beta
\end{gathered}
$$

where:

$$
\begin{gathered}
\omega_{n}^{2}=500.564+12.3 N \quad \xi_{0}=c \\
\gamma_{3}=927+28 N+151 \alpha_{1} \quad \kappa=\frac{16}{3 \pi} \alpha_{2} V_{\mathrm{ac}} V_{\mathrm{dc}} .
\end{gathered}
$$

Mathematically, $A_{\mathfrak{c}_{m}}$ is defined as the oscillation amplitude for which the equation $\frac{\mathrm{d} \Omega}{\mathrm{d} \beta}=0$ has a unique solution $\beta_{\mathrm{c}_{m}}=\frac{2 \pi}{3}$. Thus, the critical electrostatic force is deduced:

$$
\kappa_{\mathrm{c}_{m}}=\frac{4 \sqrt{2} \xi_{0}^{3 / 2} \omega_{n}^{3 / 2}}{3 \sqrt[4]{3} \sqrt{\gamma_{3}}} .
$$

The critical amplitude $A_{\mathrm{c}_{m}}$ is obtained by substituting equation (17) into equation (16b) at the point $\beta=\frac{\pi}{2}$ and multiplying by the sensing gap thickness $g_{\mathrm{d}}$ and by the coefficient of the first linear undamped mode shape $\phi_{1}$ in the middle of the beam.

$$
A_{\mathrm{c}_{m}}=1.685 \frac{h}{\sqrt{Q}} .
$$

Equation (18) represents the classical form of the critical amplitude for a Duffing resonator (only mechanical nonlinearity) [3]. It shows that the critical amplitude is only determined by the beam thickness in the direction of vibration $h$ and the quality factor $Q$ and does not depend on the beam length $l$. This information has been observed experimentally by Shao et al [22] for micromechanical clamped-clamped beam resonators using stroboscopic SEM.

Our model allows the computation of the critical amplitude when all sources of nonlinearities are included: it can be deduced in the same way as explained in the simplified case above.

$$
\begin{gathered}
A_{\mathrm{c}}=\sqrt{\theta_{1} h^{2}-\theta_{2}+\sqrt{\theta_{3}-\theta_{4} h^{2}+\theta_{5} h^{4}+\frac{\theta_{6} h^{2}}{Q}}} \\
\theta_{1}=0.003632757220621099 \\
\theta_{2}=\frac{19328 g_{\mathrm{d}}^{2}}{43375} \\
\theta_{3}=0.19856141464508456 g_{\mathrm{d}}^{4} \\
\theta_{4}=0.0032375299854832426 g_{\mathrm{d}}^{2} \\
\theta_{5}=0.000013196925023975936 \\
\theta_{6}=2.56831390855867 g_{\mathrm{d}}^{2} .
\end{gathered}
$$

We can easily check that $\lim _{g_{\mathrm{d}} \rightarrow \infty} A_{\mathrm{c}}=A_{\mathrm{c}_{m}}$.

For example, the critical amplitude of a resonator having a quality factor of $10^{4}$ designed with $100 \mathrm{~nm}$ of thickness in the direction of vibration and a sensing gap thickness of $200 \mathrm{~nm}$ is about $1.68 \mathrm{~nm}$.

$0.84 \%$ of the gap thickness is thus the restrictive amplitude in order to stay linear, which leads to a very weak signal to noise ratio and thus a low resolution.

\subsection{Pull-in}

The pull-in amplitude is the oscillation amplitude above which the resonator position becomes unstable and collapses. The dynamic pull-in is the collapse of the beam subjected to a timevarying electrostatic force, completely different from the static pull-in [13] where the electrostatic force depends only on the gap.

In the general case, pull-in can occur for hardening and softening behavior even at amplitudes lower than $A_{\mathrm{p}}$. Nevertheless, this study here is restricted to practical cases of nanoresonators which are designed with gaps and width in the direction of vibration of the same order of magnitude.

In the softening domain, the existence of an inevitable escape band (band where no other possible solution exists except pull-in [23, 24]) is very likely (even for small AC voltage) and consequently it is not wished to work in this domain. Anyway, this would mean being able to fabricate a much smaller gap than the beam width, which is of great difficulty with top-down technology.

On the other hand, hardening behavior has been easily observed in our experiments as in many others [9, 22], without pull-in occurrence, although it is theoretically possible in the general case for initial conditions outside the homoclinic manifold associated with the system. This may be explained by two facts: for our typical designs, basins of attraction of 
upper and lower stable branches are much larger than pullin attractors at points 5,6 and 7. Secondly, the ensemble of possible initial conditions in practical cases of electrical characterization is rather limited (small static displacement versus dynamic on section 2.3 , slow change of frequency ...).

Finally, in the mixed regime, the point $\mathrm{P}$ is a global bifurcation [25, 26], meaning it is the limit above which a complex dynamic behavior called homoclinic tangle [27] appears. This leads to a high sensitivity to initial conditions or the unpredictability of motion [12], which is undesirable for NEMS designers. In particular, the pull-in instability may occur at the bifurcation point $\mathrm{P}$, where the effect of the nonlinear electrostatic stiffness becomes significantly important, and where the domain of attraction of stable branches is small, making the jump of the system to these stable branches quite hard physically [12]. Consequently, and like other studies [24], we define the point $\mathrm{P}$ as the initiation of an instability domain and thus an upper bound of possible drive $\left(V_{\mathrm{p}}=\left(V_{\mathrm{ac}} V_{\mathrm{dc}}\right)_{\mathrm{p}}\right)$, beyond which dynamic pullin (characterized by a Floquet multiplier approaching unity) is likely to occur. Consequently, the pull-in domain initiation amplitude is defined as $A_{\mathrm{p}}=A\left(\frac{\pi}{2}, V_{\mathrm{p}}\right)$.

Using this criterion, we situate the initiation of the dynamic pull-in domain using the model via the transition from two to three bifurcation points as shown in figure 2(b). The third bifurcation point is situated at the phase $\beta=\frac{\pi}{2}$ which corresponds to the initiation of the mixed behavior. The latter is characterized by a slope approaching infinity at point $\mathrm{P}$ as shown in figure 2(b). Therefore, the pull-in can occur when $\frac{\mathrm{d} \Omega\left(\frac{\pi}{2}\right)}{\mathrm{d} \beta}=\frac{\mathrm{d}^{2} \Omega\left(\frac{\pi}{2}\right)}{\mathrm{d} \beta^{2}}=0$ and the $V_{\mathrm{p}}$ voltage is directly deduced.

$$
\begin{aligned}
V_{\mathrm{p}}= & \left\|\frac{3 l \pi c \omega_{n} \Delta\left(-\chi_{3}+\mu_{2} \omega_{n}^{2}\right)^{\frac{1}{2}}\left[\chi_{5}-\mu_{4} \omega_{n}^{2}\right]^{-\frac{5}{2}}}{400 \sqrt{10} \alpha_{2} C_{n 1}\left(l^{2} \cos \left[\frac{\pi}{l}\right] \sin \left[\frac{\pi l_{\mathrm{d}}}{l^{2}}\right]-\pi l_{\mathrm{d}}\right)}\right\| \\
\Delta= & 200 \chi_{5}^{2}+\mu_{4}\left(-21 \mu_{2}^{2}+200 \mu_{4}\right) \omega_{n}^{4}-30 \mu_{2} \chi_{3} \chi_{5} \\
& +2\left(6 \mu_{2} \mu_{4} \chi_{3}+5\left(3 \mu_{2}^{2}-40 \mu_{4}\right) \chi_{5}\right) \omega_{n}^{2}+9 \mu_{4} \chi_{3}^{2} .
\end{aligned}
$$

In the particular case of $V_{\mathrm{s}}=V_{\mathrm{dc}}$, the electrostatic force due to the second electrode is null and the model is similar to a resonator comprising only one electrode. For the resonator described in figure 2(a) $\left(l_{\mathrm{a}}=l_{\mathrm{d}}=200 \mu \mathrm{m}\right)$, dynamic pull-in voltage has been computed using a published formula based on an energetic analysis and validated experimentally [28], which actually gives results in good agreement with equation (20).

\subsection{Engineering optimization}

To the authors' knowledge, it is the first time that closedform expressions of the critical amplitude and the pullin domain initiation amplitude with full mechanical and electrostatic nonlinearities have been deduced thanks to the model (figure 2(b)) in the complicated case of resonator design described in figure 1. Hence, it constitutes an interesting tool to set the highest drive possible of the resonator while keeping its behavior linear. The hysteresis suppression [29] is based on the tuning of the parameter $\frac{h}{g_{\mathrm{d}}}$, which permits the enhancement of resonant sensor resolution. Thus, the rate of enhancement can be written as

$$
\Pi_{\mathrm{enh}}=\frac{A_{\mathrm{p}}}{A_{\mathrm{c}}} .
$$

In the particular case of figure 2(a), the critical amplitude is $A_{\mathrm{c}}=0.02 g_{\mathrm{d}}=15 \mathrm{~nm}$. Using the model, the hysteresis suppression is possible for $\frac{h}{g_{2}} \approx 4$ and the pull-in domain initiation amplitude is $A_{\mathrm{p}} \approx 0.6 g_{\mathrm{d}}$. Therefore, the enhancement rate of the sensor performance $\Pi_{\text {enh }}$ is around 30 .

\subsection{Numerical validation}

All the numerical simulations were carried out for the typical resonator described in figure 2(a). The equation of motion (equation $(5 a)$ ) projected on the first mode of the clamped microbeam has been integrated numerically using the fourth order Runge-Kutta algorithm at different frequencies. The frequency range was chosen from the analytical curve plot to cover the complete nonlinear frequency characteristic of the resonator. Then, the maximum steady-state displacement was extracted from each numerical simulation and the numerical points have been superimposed on the same plot with the analytical curves.

These integrations have been performed for different physical parameters giving dynamic behaviors going from slightly to extremely nonlinear. Figure 3(a) shows some comparisons in very good agreement between the numerical integration and analytical model.

Next, as the normal modes are not orthogonal with respect to the operator $w^{4} \frac{\partial^{4} w}{\partial x^{4}}$ which has been used and in order to validate the Galerkin projection, the system of five coupled nonlinear ordinary differential equations corresponding to the projections of the equation of motion (equation $(5 a)$ ) on the first five modes has been integrated numerically using the assumption of the same modal damping on each mode. As the electrostatic forces are distributed symmetrically along the beam, the antisymmetric modes 2 and 4 have no contributions to the frequency response of the resonator, and consequently the system has been simplified from five to three equations. Again, figure 3(b) shows some comparisons in good agreement between numerical integration and the analytical model; in particular, this plot shows that the contribution of the higher modes is negligible, achieving the numerical validation of the model.

\subsection{The fringing field effect}

To compute the actuation and detection capacitances, the fact has been taken into account that the geometry is far from semi-infinite plate capacitors. For weak ratios $\frac{g}{b}$, the fringing field effects are negligible. In our typical cases of ratios $\frac{g}{b}$, higher than 0.05 , they can significantly increase the value of the capacitance compared to that of the parallel plate model.

The fringing field coefficients $C_{n 1}$ and $C_{n 2}$ have been calculated using an analytical model $\left(C_{n}=1+1.9861\right.$ $\left.\left(\frac{g}{b}\right)^{0.8258}\right)$ [17]. In our case, $C_{n 1}=1.6$ and $C_{n 2}=1.5$.

These values have been validated using $3 \mathrm{D}$ COMSOL Multiphysics FE simulations. Figure 4 shows the electric potential in a cross section of the resonator described in figure 2(a) parallel to the beam motion, where the inner white rectangle represents the beam and the colored part represents the air box and the silicon bulk box respectively placed up and down from the mid-plane of the beam. In addition, the 


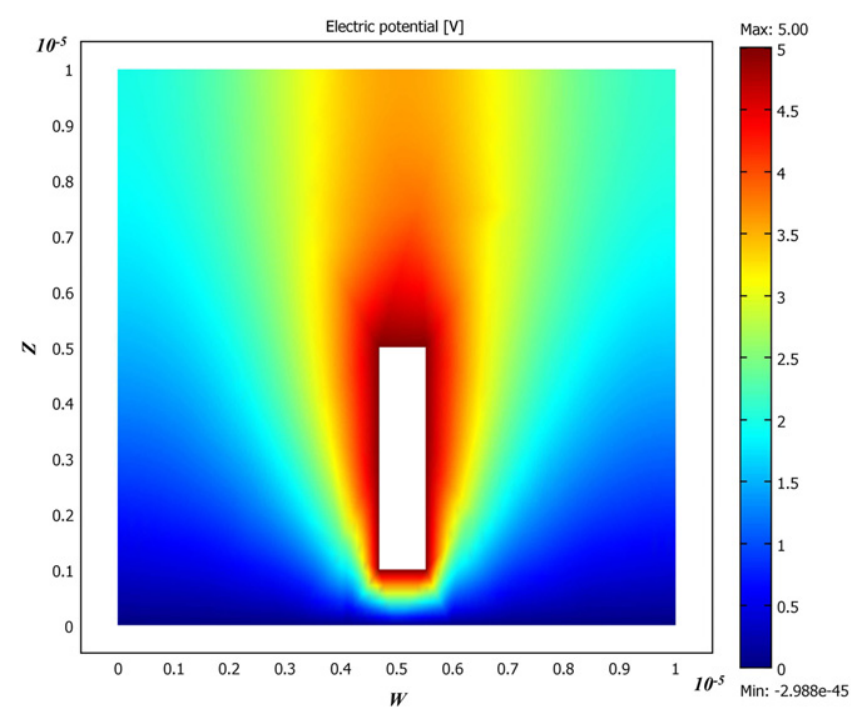

Figure 4. Fringing field effect: distribution of the electric potential in a cross section of the resonator in the plane $(W, Z)$ under $5 \mathrm{~V}$ DC voltage.

silicon oxide between the electrodes and the silicon bulk was incorporated in the 3D FE model. The quantities of electric charges $Q_{1}$ and $Q_{2}$ have been integrated numerically at each electrode in order to estimate the real capacitances $C_{1}$ and $C_{2}$, and thus the fringing field coefficients are directly deduced, $C_{n i}=\frac{C_{i}}{C_{0} i}=\frac{2 Q_{i} g_{i}^{2}}{\varepsilon_{0} b l V^{3}}$, where $g_{i}$ is the gap thickness and $V$ is the DC voltage applied to the beam as a boundary condition in the 3D FE simulations.

\section{Experimental validation}

\subsection{Manufacturing}

The fabrication starts with $200 \mathrm{~mm}$ SOI wafers $(4 \mu \mathrm{m} \mathrm{Si}$, $1 \mu \mathrm{m} \mathrm{SiO} 2$ ). The use of DUV lithography combined with a deep RIE process has allowed $500 \mathrm{~nm}$ wide gaps and lines. Some low stiffness beams have been designed, so the HF-vapor technique had to be improved to enable release and protection against in-plane sticking. A very simplified process flow is shown in figure 5 .

\subsection{Experimental characterization}

The fabricated resonators are electrostatically actuated in plane. These resonators are described in figures 2(a) and 6 and their measured quality factors are very high $\left(10^{4}-5 \times 10^{5}\right)$. As a consequence, the critical amplitude is around $15 \mathrm{~nm}$ and thus the capacitance variation is around $2 \mathrm{aF}$. Considering the low capacitance variations and the high motional resistance combined with the important parasitic capacitances, tracking the resonance peak purely electrically is really difficult. Being at the limit of electric direct measurement, a SEM set-up was developed as a first step, coupled with a real-time in situ electrical measurement using an external low noise lock-in amplifier (figure 7).

This set-up allows the simultaneous visualization of the resonance by SEM imaging (figure 6) and the motional current frequency-response measurement.

As a second step, once the resonance frequency was found, the SEM set-up was not used to allow for precise measurements and the device was placed in a vacuum chamber and measurements were performed at room temperature. The residual stress $\left(\frac{\tilde{N}}{b h}\right)$ calculated knowing the frequency shift between the natural frequency and the measured frequency is around $15 \mathrm{MPa}$ and the fringing field effect coefficients are $C_{n 1}=1.6$ and $C_{n 2}=1.5$. As shown in figure 8, the raw signal given by the lock-in amplifier shows a weak resonance peak drowned in a large background, followed by an antiresonance, both due to a large feedthrough capacitance. To get rid of this effect, a measurement is carried out with null DC voltage. In this case, the beam does not resonate, and thus no motional signal is measured. The vectorial subtraction of the two signals gives the signal purely due to the motional current, which is compared with the model results.

Considering the equivalent electrical scheme of the measurement chain (see figure 9), the output voltage generated by this system can be expressed as

$$
\begin{gathered}
V_{\text {out }}(t)=Z_{t}\left(V_{\mathrm{dc}}-V_{\mathrm{s}}\right) \frac{\mathrm{d} C_{\text {res }}}{\mathrm{d} t} \\
\frac{\mathrm{d} C_{\text {res }}}{\mathrm{d} t}=\int_{\frac{l-l_{\mathrm{d}}}{2 l}}^{\frac{l+l_{\mathrm{d}}}{2 l}} \frac{b C_{n 2} \epsilon_{0} \phi_{1}(x) a_{1}^{\prime}(t)}{\left(1-a_{1}(t) \phi_{1}(x)\right)^{2}} \mathrm{~d} x
\end{gathered}
$$

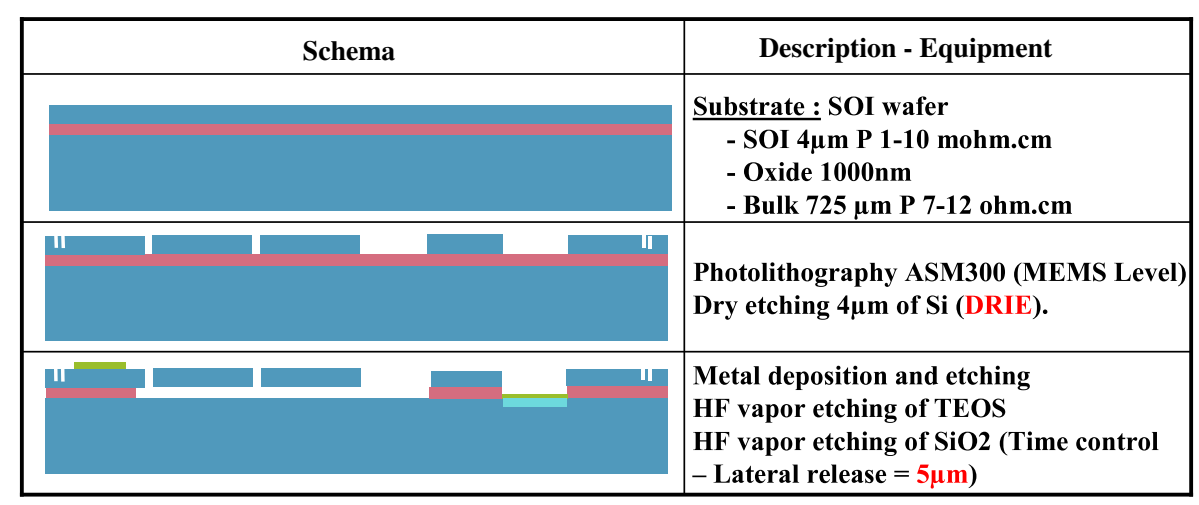

Figure 5. Simplified process flow. 

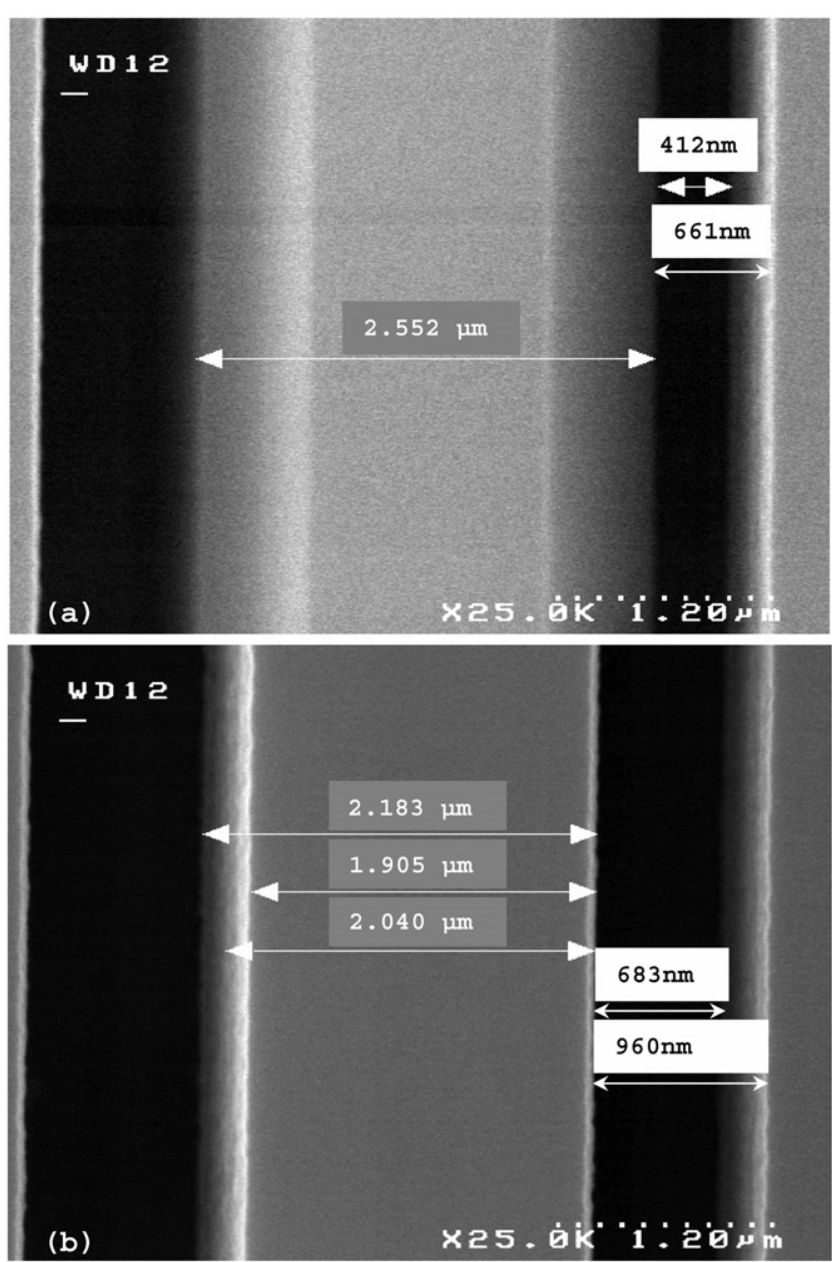

Figure 6. (a) SEM image of the resonator resonance. (b) SEM image of the resonator at rest. Dimensions: $200 \mu \mathrm{m} \times 2 \mu \mathrm{m} \times 4 \mu \mathrm{m}$. Gap: around $750 \mathrm{~nm}$.

$$
Z_{t}=\frac{Z_{\text {cable }} Z_{\text {lock in }}}{Z_{\text {cable }}+Z_{\text {lock in }}}
$$

where $V_{\mathrm{s}}$ is the DC voltage applied to the sensing electrode, $Z_{\text {lock in }}$ is the internal impedance of the lock-in amplifier and $Z_{\text {cable }}$ is the impedance of the parasitic capacitances due to the connection cables.

The output voltage was calculated analytically using the results of the reduced-order model in displacement (equation (15a)) and a Taylor series expansion of the capacitance. All results shown below were obtained with the same device (see figure 2(a)) using the same experimental conditions, and in particular at a pressure low enough that the quality factor has reached saturation. Only the bias and drive voltages may vary, as indicated on the graphs.

3.2.1. Linear case $\left(A<A_{c}\right)$. The vibration amplitude of the resonator is lower than the critical amplitude. It is paradoxically a difficult condition to obtain, as it demands a low drive, and thus the signals are very weak. A great effort has been needed on the noise and output capacitance reduction to get the peaks out of the background.

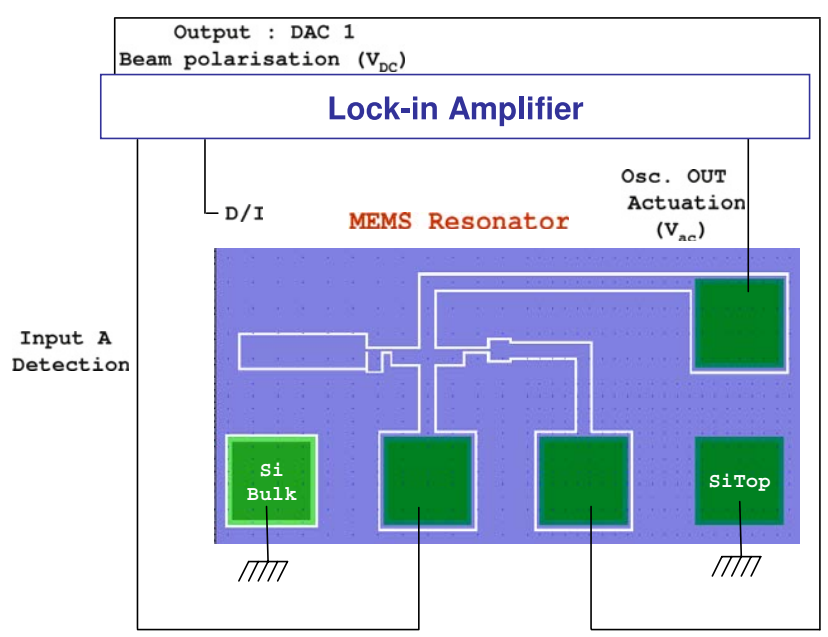

Figure 7. Connection layout for the electrical characterization.

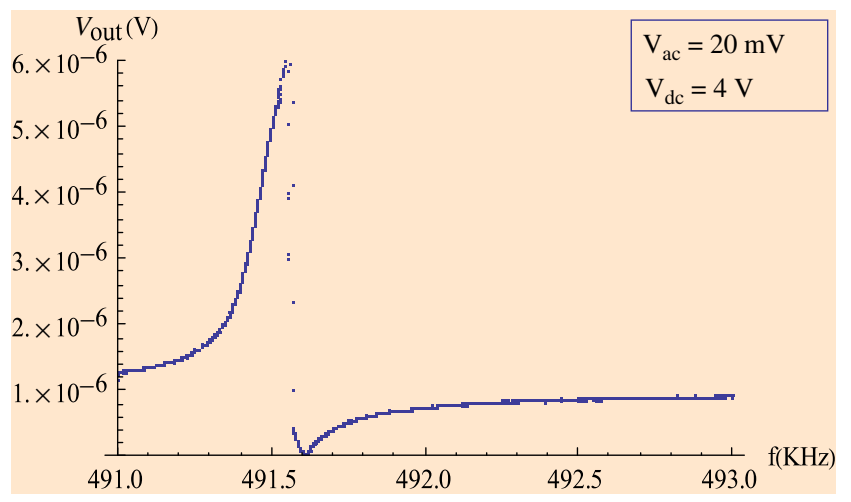

Figure 8. The raw signal given by the lock-in amplifier.

It is important to underline that all the inputs of the model are known physical parameters including the fringing field coefficients computed using the analytical formulae [17], except the quality factor $Q$ measured experimentally. So as to evaluate the model, $Q$ has been fitted using linear curves. But for NEMS design optimization, the quality factor will be computed analytically using existing models taking into account the thermoelastic damping [30], the support loss [31] and the surface loss [32] and which actually give results in good agreement with experimental measurements.

Figure 10 shows three linear peaks obtained for different values of the bias voltage $V_{\mathrm{dc}}(1 \mathrm{~V}-3 \mathrm{~V}-5 \mathrm{~V})$ and the same drive voltage. The reader will note that the loaded quality factor changes $\left(5 \times 10^{4}-23 \times 10^{3}-11 \times 10^{3}\right)$ accordingly [33]. The resonance frequency also decreases from $493 \mathrm{kHz}$ (black curve) to $490.5 \mathrm{kHz}$ (green curve) due to the negative stiffness, a phenomenon very well displayed by the model. Precisely, the effect of the negative electrostatic stiffness gives frequency shifts of $0.8 \mathrm{kHz}$ between the green and the red curves and $1.6 \mathrm{kHz}$ between the red and the black curves. Moreover, the shapes of the peaks and their predicted amplitudes using the model are in excellent correlation with the experimental measured points. Both red and black linear peaks are in the same range of oscillation amplitude $(1.8 \mathrm{~V}$ for the red peak 


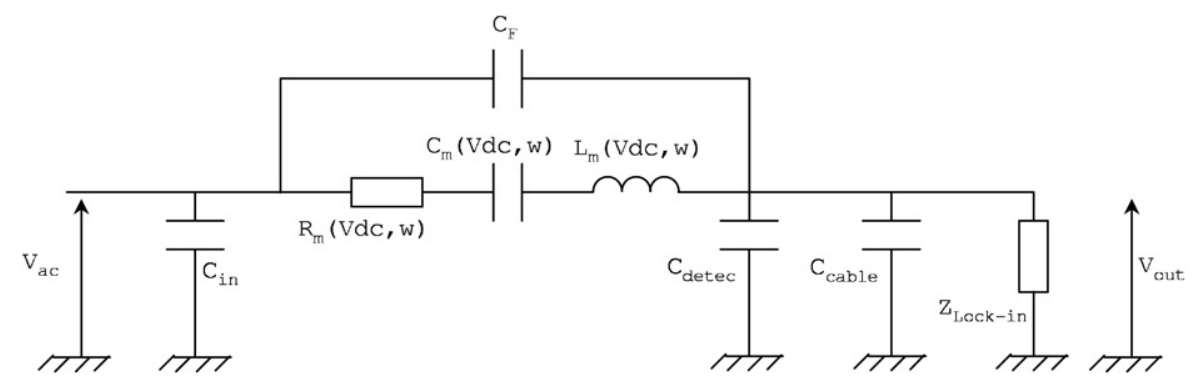

Figure 9. Equivalent electric circuit.

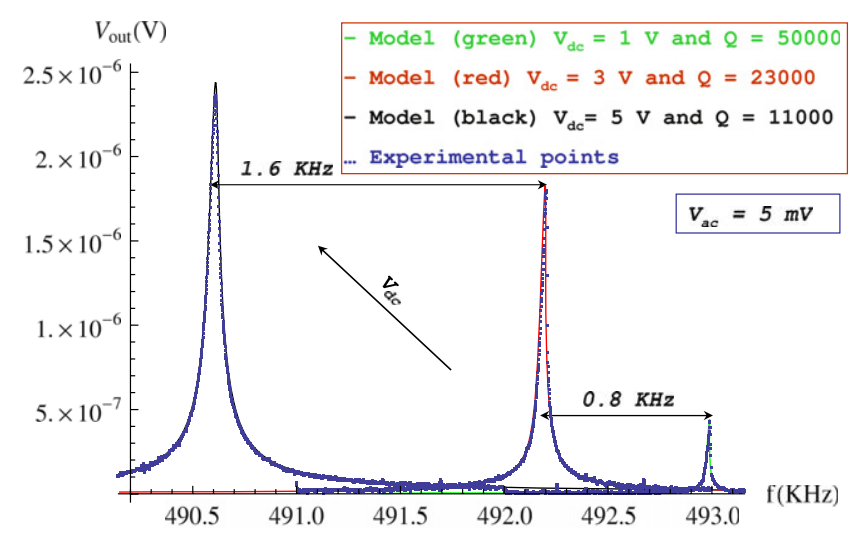

Figure 10. Measured and predicted frequency responses.

and $2.4 \mathrm{~V}$ for the black peak). However, the red peak with high quality factor $(Q=23000)$ is very close to the critical amplitude $\left(V_{\text {out }}=1.9 \mathrm{~V}\right)$, which is well in agreement with equations (18) and (19a).

3.2.2. Nonlinear case $\left(A>A_{c}\right)$. The vibration amplitude of the resonator is higher than the critical amplitude. The actuation voltage $V_{\mathrm{ac}}$ is increased from $5 \mathrm{mV}$ used for linear peaks to $20 \mathrm{mV}$ here. Figure 11 shows three nonlinear peaks, again obtained for different values of $V_{\mathrm{dc}}(1 \mathrm{~V}-3 \mathrm{~V}-5 \mathrm{~V})$. The use of the same resonator, same vacuum conditions and same bias values as in the linear case allows for the identification of the quality factors from the measurements in figure 10, assuming that no extra damping mechanism occurs. The predicted curves using the model are in very good correlation in shape and frequency shift (negative stiffness) with the measured points, although the model displays slightly higher amplitudes; the unstable jumps make it awkward to obtain precise comparison of high quality factor peaks. Indeed, it is easy to fit perfectly the experimental curves with slightly different values of width, quality factor and residual stress. This is confirmed by the fact that the ratio between the critical amplitude calculated using the model and the peak amplitude measured experimentally $\frac{V_{\text {out }}}{V_{c}}$ is around 5 for the red curve (for which the discrepancy is highest) and 3 for the green and the black curves. Consequently, the red peak is more nonlinear than the other two peaks, which is clearly shown in figure 11 from the curvature of each peak. Also, this validates the closedform expression of the critical amplitude (equation (19a)).

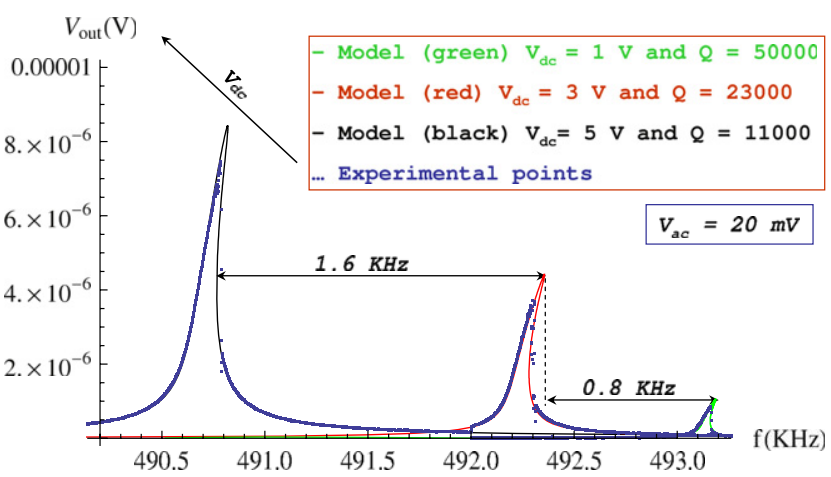

Figure 11. Measured and predicted frequency responses.

\section{Conclusions}

This work has detailed the development of an analytical model and its validation to quantitatively assess the nonlinear dynamics of M/NEMS resonators. This model includes all sources of nonlinearities, in particular of the electrostatic ones without approximation, and is based on the modal decomposition using the Galerkin procedure combined with a perturbation technique (the averaging method). This approach has been used to study doubly clamped beams, but this work will be extended in the future to other types of boundary conditions and structures. The model has been compared to numerical simulations using the Runge-Kutta algorithm and shows a very good agreement. Also, experimental validation has been performed thanks to the fabrication and electrical measurements of M/NEMS resonators, driven at different (linear and nonlinear) conditions. All parameters of the model except the quality factor are set prior to the comparison, which shows an excellent agreement in frequency, shape and amplitude, although the latter is slightly higher in the model results for highly nonlinear resonances, which will be further studied. This shows nonetheless that it can be an efficient predictive tool.

The shape of the model output (two parametric equations) has the advantage to be simple and easy to implement for M/NEMS designers. The study has notably provided for closed-form solutions of the critical amplitude including full orders of nonlinearities, as well as of the pull-in domain initiation amplitude. It has also shown how it is possible to tune some design parameters (like the ratio between the beam thickness in the direction of vibration $h$ and the detection gap 
thickness $g_{\mathrm{d}}$ ) to keep a linear behavior up to the pull-in point. The consequence of this may be a great gain in the sensors' resolution, as the resonator's carrier power is largely increased while keeping linear may prevent most of noise aliasing.

Future work will consist in showing the different behaviors predicted in this work, in validating the pull-in amplitude solution and the hysteresis suppression capability, for NEMS with a critical dimension of a few hundred nanometers. Also, the behavior and stability of the system under AC axial force will be studied, analytically as well as experimentally. The model will also allow for the study of other interesting phenomena, like parametric amplification [34-36] and noise squeezing [34].

\section{Acknowledgments}

We gratefully acknowledge financial support from the Carnot institute (Carnot Nems Project) and from the ANR (MNTEurop Project).

\section{Appendix}

$$
\begin{aligned}
& \omega_{n}^{2}=-2\left(V_{\mathrm{s}}^{2}+V_{\mathrm{dc}}^{2}\right) \frac{C_{n 2} \alpha_{2}}{R_{\mathrm{g}}} \int_{\frac{l-l_{\mathrm{d}}}{2 l}}^{\frac{l+l_{\mathrm{d}}}{2 l}} \phi_{1}^{2} \mathrm{~d} x \\
& +C_{n 1} \alpha_{2} \frac{2 V_{\mathrm{dc}}^{2}+V_{\mathrm{ac}}^{2}}{R_{\mathrm{g}}^{2}} \int_{\frac{l-l_{\mathrm{a}}}{2 l}}^{\frac{l+l_{\mathrm{a}}}{2 l}} \phi_{1}^{2} \mathrm{~d} x \\
& 4 V_{\mathrm{dc}} V_{\mathrm{s}} \frac{C_{n 2} \alpha_{2}}{R_{\mathrm{g}}} \int_{\frac{l-l_{\mathrm{d}}}{2 l}}^{\frac{l+l_{\mathrm{d}}}{2 l}} \phi_{1}^{2} \mathrm{~d} x+\lambda_{1}^{2}-N \int_{0}^{1} \phi_{1} \phi_{1}^{\prime \prime} \mathrm{d} x \\
& \mu_{1}=-2 \int_{0}^{1} \phi_{1}^{3} \mathrm{~d} x \frac{\left(1+R_{\mathrm{g}}\right)}{R_{\mathrm{g}}} \\
& \mu_{2}=\frac{1+4 R_{\mathrm{g}}+R_{\mathrm{g}}^{2}}{R_{\mathrm{g}}^{2}} \int_{0}^{1} \phi_{1}^{4} \mathrm{~d} x \\
& \mu_{3}=-\frac{2\left(1+R_{\mathrm{g}}\right)}{R_{\mathrm{g}}^{2}} \int_{0}^{1} \phi_{1}^{5} \mathrm{~d} x \\
& \mu_{4}=-\frac{2\left(1+R_{\mathrm{g}}\right)}{R_{\mathrm{g}}^{2}} \int_{0}^{1} \phi_{1}^{5} \mathrm{~d} x \\
& \chi_{2}=\left(-2 \lambda_{1}^{2} \frac{\left(1+2 R_{\mathrm{g}}\right)}{R_{\mathrm{g}}}-\frac{C_{n 1} \alpha_{2} V_{\mathrm{ac}}^{2}}{R_{\mathrm{g}}^{2}}\right) \int_{0}^{1} \phi_{1}^{3} \mathrm{~d} x \\
& +C_{n 2} \alpha_{2} \frac{\left(V_{\mathrm{dc}}^{2}-2 V_{\mathrm{dc}} V_{\mathrm{s}}+V_{\mathrm{s}}^{2}\right)}{R_{\mathrm{g}}^{2}} \int_{\frac{l-l_{\mathrm{d}}}{2 l}}^{\frac{l+l_{\mathrm{d}}}{2 l}} \phi_{1}^{3} \mathrm{~d} x \\
& -\frac{V_{\mathrm{dc}}^{2} C_{n 1} \alpha_{2}}{R_{\mathrm{g}}^{2}} \int_{\frac{l-l_{\mathrm{a}}}{2 l}}^{\frac{l+l_{\mathrm{a}}}{2 l}} \phi_{1}^{3} \mathrm{~d} x+2 N \frac{\left(1+2 R_{\mathrm{g}}\right)}{R_{\mathrm{g}}} \int_{0}^{1} \phi_{1}^{2} \phi_{1}^{\prime \prime} \mathrm{d} x \\
& \chi_{3}=-N \frac{1+4 R_{\mathrm{g}}+R_{\mathrm{g}}^{2}}{R_{\mathrm{g}}^{2}} \int_{0}^{1} \phi_{1}^{3} \phi_{1}^{\prime \prime} \mathrm{d} x \\
& +\lambda_{1}^{2} \frac{1+4 R_{\mathrm{g}}+R_{\mathrm{g}}^{2}}{R_{\mathrm{g}}^{2}} \int_{0}^{1} \phi_{1}^{4} \mathrm{~d} x \\
& -\alpha_{1} \int_{0}^{1}\left(\int_{0}^{1} \phi_{1}^{\prime 2} \mathrm{~d} x\right) \phi_{1} \phi_{1}^{\prime \prime} \mathrm{d} x
\end{aligned}
$$

$$
\begin{aligned}
& \chi_{4}=2 \alpha_{1} \frac{1+R_{\mathrm{g}}}{R_{\mathrm{g}}} \int_{0}^{1}\left(\int_{0}^{1} \phi_{1}^{\prime 2} \mathrm{~d} x\right) \phi_{1}^{2} \phi_{1}^{\prime \prime} \mathrm{d} x \\
& +2 N \frac{1+R_{\mathrm{g}}}{R_{\mathrm{g}}^{2}} \int_{0}^{1} \phi_{1}^{4} \phi_{1}^{\prime \prime} \mathrm{d} x-2 \lambda_{1}^{2} \frac{1+R_{\mathrm{g}}}{R_{\mathrm{g}}^{2}} \int_{0}^{1} \phi_{1}^{5} \mathrm{~d} x \\
& \chi_{5}=\frac{\lambda_{1}^{2}}{R_{\mathrm{g}}^{2}} \int_{0}^{1} \phi_{1}^{6} \mathrm{~d} x-\frac{N}{R_{\mathrm{g}}^{2}} \int_{0}^{1} \phi_{1}^{5} \phi_{1}^{\prime \prime} \mathrm{d} x \\
& -\alpha_{1} \frac{1+4 R_{\mathrm{g}}+R_{\mathrm{g}}^{2}}{R_{\mathrm{g}}^{2}} \int_{0}^{1}\left(\int_{0}^{1} \phi_{1}^{\prime 2} \mathrm{~d} x\right) R_{\mathrm{g}}^{2} \alpha_{1} \phi_{1}^{3} \phi_{1}^{\prime \prime} \mathrm{d} x \\
& \chi_{6}=2 \alpha_{1} \frac{1+R_{\mathrm{g}}}{R_{\mathrm{g}}^{2}} \int_{0}^{1}\left(\int_{0}^{1} \phi_{1}^{\prime 2} \mathrm{~d} x\right) \phi_{1}^{4} \phi_{1}^{\prime \prime} \mathrm{d} x \\
& \chi_{7}=-\frac{\alpha_{1}}{R_{\mathrm{g}}^{2}} \int_{0}^{1}\left(\int_{0}^{1} \phi_{1}^{\prime 2} \mathrm{~d} x\right) \phi_{1}^{5} \phi_{1}^{\prime \prime} \mathrm{d} x \\
& v=-\frac{1}{2}\left(C_{n 1} \alpha_{2}\left(V_{\mathrm{ac}}^{2}+2 V_{\mathrm{dc}}^{2}\right) \int_{\frac{l-l_{\mathrm{a}}}{2 l}}^{\frac{l+l_{\mathrm{a}}}{2 l}} \phi_{1} \mathrm{~d} x\right) \\
& +C_{n 2} R_{\mathrm{g}}^{2} \alpha_{2}\left(V_{\mathrm{dc}}^{2}-2 V_{\mathrm{dc}} V_{\mathrm{s}}+V_{\mathrm{s}}^{2}\right) \int_{\frac{l-l_{\mathrm{d}}}{2 l}}^{\frac{l+l_{\mathrm{d}}}{2 l}} \phi_{1} \mathrm{~d} x \\
& \zeta_{0}=4 V_{\mathrm{ac}} V_{\mathrm{dc}} C_{n 1} \alpha_{2} \int_{\frac{l-l_{a}}{2 l}}^{\frac{l+l_{\mathrm{a}}}{2 l}} \phi_{1} \mathrm{~d} x \\
& \zeta_{1}=-4 V_{\mathrm{ac}} V_{\mathrm{dc}} C_{n 1} \alpha_{2} \int_{\frac{l-l_{\mathrm{a}}}{2 l}}^{\frac{l+l_{\mathrm{a}}}{2 l}} \phi_{1}^{2} \mathrm{~d} x \\
& \zeta_{2}=2 V_{\mathrm{ac}} V_{\mathrm{dc}} C_{n 1} \alpha_{2} \int_{\frac{l-l_{\mathrm{a}}}{2 l}}^{\frac{l+l_{\mathrm{a}}}{2 l}} \phi_{1}^{3} \mathrm{~d} x \\
& \zeta_{3}=-V_{\mathrm{ac}}^{2} C_{n 1} \alpha_{2} \int_{\frac{l-l_{\mathrm{a}}}{2 l}}^{\frac{l+l_{\mathrm{a}}}{2 l}} \phi_{1} \mathrm{~d} x \\
& \zeta_{4}=V_{\mathrm{ac}}^{2} C_{n 1} \alpha_{2} \int_{\frac{l-l_{\mathrm{a}}}{2 l}}^{\frac{l+l_{\mathrm{a}}}{2 l}} \phi_{1}^{2} \mathrm{~d} x \\
& \zeta_{5}=-\frac{1}{2} V_{\mathrm{ac}}^{2} C_{n 1} \alpha_{2} \int_{\frac{l-l_{\mathrm{a}}}{2 l}}^{\frac{l+l_{\mathrm{a}}}{2 l}} \phi_{1}^{3} \mathrm{~d} x .
\end{aligned}
$$

\section{References}

[1] Huang X M H, Zorman C A, Mehregany M and Roukes M L 2003 Nature 421496

[2] Peng H B, Chang C W, Aloni S, Yuzvinsky T D and Zettl A 2006 Phys. Rev. Lett. 97087203

[3] Postma H W C, Kozinsky I, Husain A and Roukes M L 2005 Appl. Phys. Lett. 86223105

[4] Roukes M L 2001 Nanoelectromechanical systems face the future Phys. World 14 25-31

[5] Robins W P 1982 Phase Noise in Signal Sources Peter Peregrinus LTD

[6] Juillard J, Alain B, Avignon A, Hentz S, Kacem N and Colinet E 2008 Proc. Int. Conf. IEEE Sensors (Italie: Lecce) pp 510-3

[7] Kaajakari V, Koskinen J K and Mattila T 2005 IEEE Trans. Ultrason. Ferroelectr. Freq. Control 52 2322-1

[8] Tilmans H A and Legtenberg R 1994 Sensors Actuators A 45 67-84

[9] Gui C, Legrenberg R, Tilmans H A, Fluitman J H J and Elwenspoek M 1998 J. Microelectromech. Syst. 7 122-7

[10] Kozinsky I, Postma H W Ch, Bargatin I and Roukes M L 2006 Appl. Phys. Lett. 88253101 
[11] Najar F, Choura S, Abdel Rahman E M, El-Borgi S and Nayfeh A H 2006 J. Micromech. Microeng. 16 2449-57

[12] Nayfeh A H, Younis M I and Abdel-Rahman E M 2007 J. Nonlinear Dyn. 48 153-63

[13] Osterberg P M and Senturia S D 1997 J. Microelectromech. Syst. 6 107-18

[14] Abdel-Rahman E M, Younis M I and Nayfeh A H 2002 J. Micromech. Microeng. 12 759-66

[15] Younis M I, Abdel-Rahman E M and Nayfeh A H 2003 J. Microelectromech. Syst. 12 672-80

[16] Landau L D and Lifshitz E M 1986 Theory of Elasticity 3rd edn (Oxford: Butterworth-Heinemann)

[17] Nishiyama H and Nakamura M 1990 IEEE Trans. Compon. Hybrids Manuf. Technol. 13 417-23

[18] Husain A, Hone J, Postma H W Ch, Huang X M H, Drake T, Barbic M, Scherer A and Roukes M L 2003 Appl. Phys. Lett. 83 1240-2

[19] Nayfeh A H 1981 Introduction to Perturbation Techniques (New York: Wiley)

[20] Nayfeh A H and Mook D T 1979 Nonlinear Oscillations (Physics and Applied Mathematics: A Wiley-Interscience Series of Texts) 1st edn, Monographs and Tracts (New York: Wiley)

[21] Kaajakari V, Mattila T, Lipsanen A and Oja A 2005 Sensors Actuators A 120 64-70

[22] Shao L C, Palaniapan M and Tan W W 2008 J. Micromech. Microeng. 18065014

[23] Thompson J M T and Stewart H B 2001 Nonlinear Dynamics and Chaos (New York: Wiley)
[24] Ouakad H M and Younis M I 2008 Proc. Int. Mechanical Engineering Congress and Exposition (Boston, MA)

[25] Lenci S and Rega G I 2006 J. Micromech. Microeng. 16 390-401

[26] Gottlieb O and Champneys A R 2005 IUTAM Symp. on Chaotic Dynamics and Control of Systems and Processes in Mechanics Solid Mechanics and Its Applications vol 122, ed G Rega and F Vestroni (Berlin: Springer) pp 47-57

[27] Nayfeh A H and Balachandran B 1995 Applied Nonlinear Dynamics (New York: Wiley)

[28] Fargas-Marques A, Casals-Terré J and Shkel A M 2007 J. Microelectromech. Syst. 16 1044-53

[29] Kacem N, Hentz S, Fontaine H, Nguyen V, Robert P, Legrand B and Buchaillot L 2008 Proc. Int. Conf. Nanotech. (Boston, MA) vol 3, pp 619-22

[30] Lifshitz R and Roukes M L 2000 Phys. Rev. B 61 5600-9

[31] Hao Z, Erbil A and Ayazi F 2003 Sensors Actuators A 109 156-64

[32] Yang J, Ono T T and Esashi M 2002 J. Microelectromech. Syst. $11775-83$

[33] Sazonova V 2006 A tunable carbon nanotube resonator PhD Dissertation Cornell University, Ithaca, New York

[34] Rugar D and Grütter P 1991 Phys. Rev. Lett. 67 699-702

[35] Carr D W, Evoy S, Sekaric L, Craighead H G and Parpia J M 2000 Appl. Phys. Lett. 77 1545-7

[36] Carr D W, Evoy S, Sekaric L, Craighead H G and Parpia J M 1999 Appl. Phys. Lett. 75 920-2 